\title{
Fortalecimiento de la Ciudadanía y Transformación Social: Área de Encuentro entre la Psicología Política y la Psicología Comunitaria
}

\section{Strengthening Citizenship and Social Transformation: Cross Roads Between Political Psychology and Community Psychology}

\author{
Maritza Montero \\ Universidad Central de Venezuela
}

\begin{abstract}
En este artículo se enfocan las relaciones entre psicología comunitaria (PC) y psicología política (PP), centrándose en los aportes que la PC hace a la PP y en los aspectos en los que ambas ramas de la psicología coinciden. Se parte de la idea de transformación social presente en ambas subdisciplinas psicológicas y en el objetivo central de la $\mathrm{PC}$, como se reconoce en la literatura latinoamericana y anglosajona, señalando su carácter móvil y la importancia de la participación y el compromiso en su logro. Se discuten las nociones de poder y fortalecimiento, señalándose la perspectiva simétrica del poder generada en América Latina, que muestra su influencia positiva en las transformaciones comunitarias. Se describen otros aportes de la PC a la PP, tales como la perspectiva ética, y relacionada con ella, la necesidad de la sensibilización de agentes externos, en paridad con agentes internos, la perspectiva liberadora y el uso de herramientas metodológicas compartidas entre ambas ramas de la psicología. Se concluye reiterando la condición política de la PC y su complementariedad con la PP.
\end{abstract}

Palabras clave: psicología comunitaria, psicología política, interrelación

\begin{abstract}
This paper deals with the relationships between community psychology (CP) and political psychology (PP), focusing on the contributions of the former to PP and on aspects that are common to both branches of psychology. Beginning with the idea of social transformation, present in both psychological subdisciplines and a central objective for Latin American and Anglo-Saxon PC, the dynamic condition of transformation is argued. Notions of power, strengthening, and empowerment are discussed, pointing out the symmetrical perspective of power generated in Latin America, whose positive influence is demonstrated in communal transformations. Other contributions are discussed, such as the ethical perspective and, related to it, the necessity of working in the sensitization of external agents as well as internal agents, the liberating perspective and the shared methodological tools used by PC and PP. It concludes by demonstrating the political condition of PC and its complementary relationship with PP.
\end{abstract}

Keywords: community psychology, political psychology, interrelationships

Se ha dicho que entre las características de la psicología comunitaria (PC) generada en América Latina está el tener un carácter político (Lane \& Sawaia, 1991; Montero, 1998, 2004, 2006a; Prilleltensky, 2008), ya que el desarrollo y fortalecimiento de las comunidades como colectivos e individualmente, en las personas de algunos (a veces muchos, a veces pocos) de sus integrantes, es una forma de desarrollo y fortalecimiento de la sociedad civil y la ciudadanía. Pero no es frecuente leer en los textos de psicología política (PP) aspectos relacionados con esa función y condición política de la PC, si bien se habla, y mucho, del rol político de la comunidad.

Maritza Montero, Doctorado en Psicología, Facultad de Humanidades y Educación, Universidad Central de Venezuela, Caracas, Venezuela.

Este artículo se basa, parcialmente, en una conferencia dictada por la autora en el Quincuagésimo Aniversario de la Escuela de Psicología de la Pontificia Universidad Católica de Chile, en noviembre de 2008.

La correspondencia relativa a este artículo debe ser dirigida a Maritza Montero, Apartado 80394, Concresa, Caracas, 1080-A, Venezuela. E-mail: mmonteroster@gmail.com 
En este artículo me referiré a ese lugar de encuentro entre PC y PP que surge en las comunidades y a los aportes que la primera de estas subdisciplinas hace a la segunda; aportes que deben ser vistos como actos políticos compartidos, pues, en la medida en que una comunidad hace oír sus voces en los espacios públicos y reclama y ejerce sus derechos sociales, políticos y humanos, se está actuando desde, para y con la comunidad y a la vez se realizan acciones políticas, con consecuencias tanto comunitarias como para la sociedad en general.

Cuando se habla desde la politología o desde la PP de la comunidad, se lo hace en tanto ella es una institución pública y, por lo tanto, un espacio político. La PC, a su vez, introduce la perspectiva psicosocial $\mathrm{y}$, en algunas de sus variantes, la perspectiva de la salud o la del ambiente (e.g., salud mental en la comunidad, psicología social de la salud, psicología ambiental comunitaria). Ambas miradas son necesarias y, además, complementarias.

La PC comenzó a estructurarse en América Latina a partir de la crítica de la psicología social existente en la región, debido a su incapacidad para responder a los urgentes problemas sociales presentes en el continente. Se quería hacer una psicología social que fuese socialmente sensible, que respondiese a los problemas de nuestras sociedades y, en busca de ese objetivo, se partió de la necesidad de lograr una práctica, y sus correspondientes análisis e interpretaciones, que incidiese significativamente en su entorno y que tomase en cuenta el contexto y las colectividades y no solo a los individuos aislados. Se habló, y se habla, de transformación y cambio seguidos del calificativo social.

A mediados del siglo pasado ya la PP en nuestra América tenía como antecedente una larga historia de ideas psicopolíticas (Montero, 1986, 1987), que llegaba a los orígenes republicanos de las naciones que hoy constituyen el bloque latinoamericano, además de unos 15 años de práctica. Si bien había en aquellos inicios académicos de la PP mucho de la aceptación acrítica de los modos de hacer y de pensar la política desde la psicología, a la usanza anglosajona, francesa y alemana (e.g., Eysenck, 1960/1964;
Holzkamp, 1985; Lane \& Sears, 1964/1967; Lasswell, 1930; Lazarsfeld, Berelson \& Gaudet, 1944), ya desde los 60 se buscaba trabajar sobre las raíces históricas de fenómenos tales como las identidades sociales y culturales y su relación con la conducta social (e.g., Díaz Guerrero, 1972; Gissi, 1987; Montero, 1984; Salazar, 1983). Y para fines de los 70 se había comenzado a hablar, desde la PP y desde la naciente PC, de la transformación social y del cambio social.

\section{La Transformación Social: Tarea Comunitaria y Política}

Para la PC la transformación social aparece como un objetivo central. Hacer una psicología social socialmente sensible significaba, entonces, transformar muchas cosas: el hábitat, el modo de vida, la concepción de sí mismos/as, en tanto personas viviendo en una sociedad y formando parte de una comunidad, y la misma comunidad, pues, al transformarla, también los/as transformadores/as devenían otros y otras.

Si revisamos literatura reciente en PC, encontraremos que hay una constante preocupación relativa a la capacidad, necesidad y posibilidad de cumplir ese objetivo (Montero, 2008; Reich, Riemer, Prilleltensky \& Montero, 2007; Smith, 2006). Tal preocupación muestra la vigencia del objetivo, así como su dificultad y también la incertidumbre respecto de su alcance. Aquí caben dos preguntas que como psicólogas y psicólogos comunitarios/as debemos hacernos: ¿Es la transformación social algo que podamos alcanzar una vez para después pasar a otra cosa? ¿Es definitiva la transformación? Ciertamente no es así. No se trata de cambios o modificaciones únicos, desligados de las circunstancias, siempre complejas, en las cuales se producen. Tampoco son definitivos porque ese carácter complejo de toda sociedad humana, su condición dinámica, genera una constante variación en la sociedad. La experiencia enseña que transformar algo es solo haber logrado un hito en un camino y cada logro transformador muestra que hay aún mucho camino por andar.

Para la PC, tanto en América Latina como en otros lugares del mundo, es muy importante que, al perseguir una transfor- 
mación en una comunidad, esta se dé a través de la participación y el compromiso de los grupos organizados y de las personas interesadas en una comunidad (e.g., Irizarry \& Serrano-García, 1979; Lane \& Sawaia, 1991, 1995; López-Sánchez \& Serrano-García, 1995; Montero, 2006a; Montero \& Varas Díaz, 2007; Reich et al., 2007; Sánchez \& Wiesenfeld, 1995). Esta necesidad, entonces, no nace de una teoría, aun cuando ella sea utilizada y generada también en el proceso de actuar y reflexionar para lograr los objetivos específicos (Montero, 2009). Nace de necesidades sentidas por la comunidad, además de las necesidades normativas que puedan estar relacionadas con ellas y que suelen ser introducidas desde los programas derivados de políticas públicas para las comunidades. Por lo tanto, promover y lograr esa participación comprometida es un objetivo inmediato dentro de la finalidad más amplia de la transformación (Hernández, 1996; Montero, 1996). Así, participar supone una multiplicidad de acciones que se reflejan tanto en las relaciones y el espacio privado, como en el espacio colectivo de la comunidad y en el espacio colectivo-público de la sociedad en la cual se encuentra esa comunidad, porque parte de los actos realizados van a ocurrir en el espacio de la junta o del consejo comunal, de la parroquia, el municipio, el departamento, la ciudad, el Estado y la nación, simultáneamente.

\section{Poder y Fortalecimiento}

\section{Sobre el Poder}

Otro aspecto compartido entre PC y PP es la preocupación, investigación y acción respecto de las manifestaciones del poder, ya que el poder puede ejercer su influencia tanto desde fuera de la comunidad y hacia ella, como generarla dentro de la misma comunidad, manifestándose asimétricamente para generar formas de opresión y desigualdad (Montero, 2003a; Nelson \& Prilleltensky, 2005; Prilleltensky, 2008; Serrano-García \& López-Sánchez, 1994; Zambrano, 2007) o constituyendo un aspecto fundamental en el fortalecimiento de la comunidad y en la consecución de sus objetivos de transformación.
Una respuesta al problema del poder ha sido creada desde la PC por Serrano-García y López-Sánchez (1994). Estos autores entienden que para hacer algo es necesario tener algún poder, por lo cual, a partir de sus experiencias en Puerto Rico, reconceptualizan la noción de poder, definiéndolo desde una perspectiva simétrica, en el sentido de que en ambos polos de toda relación hay poder, solo que las formas de esos poderes pueden ser diferentes, lo cual significa que pueden manifestarse en formas distintas de competir por recursos desigualmente distribuidos. La definición de poder de Serrano-García y López-Sánchez, muy distinta de las que encontramos en la mayoría de los autores, considera que el poder es una relación social, "una interacción personal o indirecta, y cotidiana, en la cual las personas manifiestan sus consensos sociales y las rupturas entre su experiencia y su conciencia" (1994, p. 178).

La idea de la simetría en el poder permite hallar sus expresiones en todos los sujetos de la relación, lo cual, a su vez, permite generar formas de deliberación, negociación, concertación y oposición, aun cuando las fuerzas, así como los recursos que un polo de poder puede controlar y que son deseados por el otro, sean desiguales. Esa concepción permite comprender por qué, aun dentro de las más crueles dictaduras, es posible para los oprimidos superar esa situación mediante acciones que ejercen formas de poder que no corresponden a la lógica del opresor.

Eso nos lleva a considerar que tan importante como usar el poder que se tiene y que se puede expresar en modos no esperados es saber que eso puede hacerse y que las personas que buscan ciertos recursos a los cuales tienen derecho, pero que les son negados, necesitan desnaturalizar la concepción que los presenta como totalmente indefensos, débiles e incapaces. Para luchar contra el poder opresor es necesario usar el poder liberador, que reconoce al otro como igual, no como superior.

Tal concepción es necesaria por cuanto el poder asimétrico y las formas de opresión y desigualdad que de él derivan pueden estar presentes en todas partes. Esa simetría en la diversidad que se propone para la PC 
dista de ser un logro a mantener; es más bien un reto constante para los procesos de organización y fortalecimiento de las comunidades (Montero, 2003a, 2006a; Zambrano, 2007; Zimmerman, 2000). Este aspecto es una contribución para la PP, pues el fortalecimiento y la idea de poder simétrico y su uso mediante estrategias diferentes en una relación de enfrentamiento, por el disfrute de recursos asimétricamente distribuidos, trascienden el campo comunitario y proporcionan una explicación estratégica para la transformación social.

\section{El Fortalecimiento}

La respuesta a la asimetría sufrida y a la simetría ignorada, particularmente en el caso de las comunidades pobres en recursos económicos -aspecto que constituye una de las áreas de mayor aplicación de la PC-, ha sido la generación casi simultánea de dos conceptos equivalentes: el de empowerment, creado en Estados Unidos por Julian Rappaport (1981) y el de fortalecimiento o potenciación, surgido al final de las década de los 70 en varios países de América Latina (México, Panamá, Puerto Rico, Venezuela), pero que no fue objeto de sistematización y publicación sino hasta fines de los 80 , aunque sí fue utilizado en trabajos de tesis de licenciatura y de postgrado (Campos, 1978; Patiño Pompa, 1985; Patiño Pompa \& Millán Aguilar,1979; Santi, Silva \& Colmenares, 1978). Ese uso práctico sin una pronta formalización probablemente haya contribuido a cierta confusión. En 2003 di la siguiente definición de fortalecimiento, basada en mi praxis comunitaria, que volví a incluir en otra obra en 2006:

Proceso mediante el cual los miembros de una comunidad o un grupo -miembros de grupos organizados dentro de esa comunidad o personas interesadas en promover y lograr un cambio respecto de alguna circunstancia que afecta a esa comunidad o grupo- desarrollan conjuntamente capacidades y recursos para controlar su situación de vida (en un momento específico); actuando de manera comprometida, consciente y crítica, para lograr la transformación de las condiciones que juzgan negativas o que deben ser modificadas según sus necesidades y aspiraciones, transformándose al mismo tiempo, a sí mismos. (Montero 2003a, p. 72)

En 1980 Rappaport, en su conferencia presidencial de la División 27 de la American Psychological Association en Montreal (Rappaport, 1981/2002), dijo que el móvil de la PC "debería ser ampliar las posibilidades de que la gente pueda controlar sus vidas" (Rappaport, 2002, p. 135) y esa fue la función que adjudicó al concepto de empowerment. En 1987 lo definía como "un mecanismo por el cual gente, organizaciones y comunidades ganan dominio sobre sus asuntos" ( $p$. 122). En 1993 Riger agregó que esa noción, así definida, incluía tanto un sentido personal de control como preocupación por la influencia social, el poder político y los derechos legales. Zimmerman (2000, pp. 5, 7) da una definición más operativa:

El empowerment psicológico incluye creencias acerca de la propia competencia y eficacia, y una voluntad de involucrarse en actividades para ejercer control en el ambiente social y político (...) Es un constructo que integra percepciones de control personal con conductas para ejercer control.

Originalmente, es una noción de carácter individual pero, cuando se expandió mundialmente, otra ha sido su historia. Pocas veces un concepto se ha hecho tan popular tan rápidamente, pero no se queda en lo personal: en muchos lugares de América Latina, España y Portugal se adoptó el barbarismo empoderamiento pero, al analizar la práctica así llamada, encontramos o fortalecimiento o viejas prácticas institucionalistas. Se pasa a hablar de grupos, comunidades y poblaciones "empoderadas", pero lo que se hace no corresponde a lo definido por el creador y seguidores del término $\mathrm{em}$ powerment. Actualmente hay una interesante discusión crítica al respecto (Acosta Pérez et al., 2007; Fuks, 2007; Montero, 2007a; Vázquez, 2004; Zambrano 2007). Citaré la crítica que hace Vázquez (2004) del uso del concepto y del término empowerment y de 
su castellanización (empoderamiento), Este autor dice que su utilización "muchas veces indiscriminada" no ha considerado sus limitaciones, tales como "su tendencia a lo individual, su afinidad con el poder como posesión y sus raíces culturales" (p. 50), lo cual coincide con la crítica de Riger (1993). Vásquez (2004) propone, entonces, el término refortalecimiento que, como el de fortalecimiento, nace de su experiencia de trabajo comunitario. Ese nuevo concepto supone "comprender las debilidades como fortalezas", ubicándose "no donde lo social forma parte de lo personal, sino donde lo personal es político y donde el sujeto es su contexto" (p. 45). Es decir, es parte de la comunidad, que es un ente político, pues es parte del espacio público.

Esta respuesta de la PC es de orden político y, unida a otras metas comunitarias, juntas pueden constituir una propuesta psicopolítica de carácter práxico, que puede resumirse en los siguientes puntos:

- Mejorar y transformar la calidad de vida de las comunidades. El primer verbo indica las formas paliativas, el segundo supone acciones más profundas. Debo confesar que no estoy segura de que haya una clara diferencia entre una y otra forma de acción, aunque prefiero la segunda, ni de que a corto plazo podamos obtener respuestas precisas. La satisfacción se expresa de múltiples formas y es cualidad humana el desear siempre algo más.

- La formación de ciudadanía mediante la incorporación de personas interesadas en cada comunidad (y cada vez interesar a más personas), a la toma de decisiones y a las acciones de transformación. Esto supone la necesidad de sensibilizar e informar a todas aquellas personas que forman la comunidad sobre lo que se lleva a cabo y sobre las metas a alcanzar y su repercusión en la comunidad.

- Énfasis en los valores éticos y en las conductas derivadas de ellos, a lo cual está unido el desarrollo de conciencia social, lograda mediante lo que Paulo Freire (1970/1997) denominó hace más de medio siglo como concientización: proceso de base crítica que permite romper con la naturalización e ideologización que llevan a aceptar lo que afecta negativamente, y aun a pensar que no puede haber nada distinto, y a denunciar, rechazar y actuar para eliminar las formas de injusticia social y de exclusión.

- El compromiso social que, como ya es bien sabido, está ligado a la participación, con la cual tiene una relación directa. La teoría de la participación-compromiso desarrollada por la PC nos señala la relación directa y recíproca existente entre ambos, de tal manera que en una construcción ideal del hombre y la mujer políticos de profesión se debería pedir como perfil para ocupar cargos gubernamentales esa conjunción: la acción participativa comprometida y el compromiso de participar, es decir, de hacer para transformar.

- Énfasis en la perspectiva liberadora, tanto en su concepción freiriana como en el desarrollo de la tendencia generada a partir de la obra de Martín-Baró (1986) en El Salvador, pero para América Latina, y actualmente en desarrollo en los cinco continentes (Flores Osorio, 2009; Montero, 2000, 2004, 2005, 2006b, 2007b, 2009; Nelson \& Prilleltensky, 2005; Watts, Griffith \& Abdul-Adil, 2003).

\section{Sobre las Políticas Públicas, la Comunidad y la Intervención Psicosocial Política y Comunitaria}

Otro aporte de la PC al campo político está dirigido a la integración de actores sociales de manera armoniosa, eficiente, respetuosa, consciente, reflexiva y participativa. Esto es fundamental para la relación entre políticas públicas que están dirigidas a las comunidades, actores intermedios, tales como los técnicos, las ONGs, las universidades y las organizaciones, y grupos comunitarios. Este es punto álgido, pues la relación entre esos tres grupos de actores sociales con tres niveles de acción es cuando menos ardua y su ausencia es responsable de lo que podríamos llamar desencuentro político, con el fracaso o limitación de esas políticas.

La falta de sensibilización, familiari- 
zación y preparación (todas estas acciones propuestas por la PC) de los funcionarios encargados de llevar a cabo los programas y proyectos derivados de las políticas públicas parece tener el carácter de pandemia. Rodríguez-Ferreyra (2009) analiza en Uruguay los efectos debilitantes del tipo de relación que se produce entre funcionarios, profesionales y técnicos intermedios (e.g., investigadores académicos), personas interesadas y grupos organizados dentro de una comunidad, en la ejecución de políticas, tales como las relativas a la educación preescolar en su país. Su trabajo denuncia cómo se termina reduciendo la participación de la comunidad beneficiaria de algún programa a las tareas de limpieza y preparación de alimentos, sin posibilidad de opinar ni decidir. En Estados Unidos Shinn (2007) resume en una metáfora que colocó como parte del título de un artículo lo duro que puede ser trabajar con funcionarios estatales: "bailar un vals con un monstruo". Experiencias igualmente difíciles las viven colegas europeos que se quejan de la incomunicación entre los técnicos de las organizaciones gubernamentales y los especialistas universitarios; sin mencionar que a la comunidad muchas veces, en muchos países del mundo, no se le dirige la palabra, no se le pide opinión. En algunos de nuestros países podríamos agregar otras metáforas, amén de símiles y alegorías entre otras figuras retóricas. Y no solo se trata de la incomunicación; otro problema no menos importante es el tiempo de implementación de un proyecto o el súbito abandono del mismo al haber cambios políticos o decisiones de adjudicar el presupuesto a otras actividades.

Es necesario, entonces, lograr un lenguaje común comprensible para todos; comprensible para los políticos profesionales y para la ciudadanía interesada y las comunidades organizadas; explorar las necesidades sentidas y, cuando ellas no están incluidas en la política de Estado, informar y convencer a quienes las conciben sobre las urgencias existentes en la población; superar los prejuicios y estereotipos existentes en esos funcionarios y también en los técnicos y especialistas provenientes de universidades y de otras organizaciones intermedias, así como los que tienen las personas en las comunidades. Esta es una tarea pendiente tanto para la PC como para la PP, las cuales ya con cierta frecuencia son llamadas a colaborar en políticas públicas.

La ausencia de consulta o el desvío de los objetivos de las políticas sociales definidos por los técnicos -entre los cuales puede haber psicólogas y psicólogos- se debe en parte a los temores que, en el caso de muchos países latinoamericanos, suscita una tradición de Gobiernos que, en nombre de los pobres y de una nueva era, ejercen formas de opresión y generan formas de clientelismo que aumentan las desigualdades y mantienen la pobreza. Asimismo, los proyectos generados en instituciones tanto estatales como no gubernamentales, bajo el signo de la técnica, pueden cometer verdaderos atentados a la economía, a la ecología, a la historia y al respeto de los ciudadanos. Necesitamos, entonces, lograr entendimiento y respeto mutuo, borrar los prejuicios y lograr una labor conjunta, de manera participativa y comprometida. Una propuesta formulada en ese sentido es descrita por Arango Cálad (2006) en Cali, Colombia, quien la denomina política pública de la convivencia, al crear una Red de Promoción del Buen Trato y de Prevención de Violencia Intrafamiliar en esa ciudad, cuya base es la afectividad positiva. La figura del consorcio social es otra de las respuestas a este reto. El consorcio social es una forma de organización cuya base está en la comunidad, pero que incorpora a técnicos, especialistas y organismos del Estado ejecutores de políticas públicas, con distintos niveles de participación y posibilidad de un manejo eficiente y bien equilibrado de conocimientos, recursos e intereses comunitarios, más beneficios políticos. En esa figura el Estado provee los fondos provenientes de un programa de la política en cuestión, los técnicos y profesionales especializados dirigen el trabajo que se realizará para la comunidad (e.g., construcción de viviendas, obras sanitarias, vialidad o agricultura) y la organización comunitaria manifiesta sus necesidades, toma decisiones, vigila el cumplimiento satisfactorio de los planes en los cuales intervienen los tres sectores y analiza logros obtenidos, pudiendo añadir otros objetivos (nuevamente discutidos conjuntamente), además de pedir y rendir cuentas. 


\section{Las Herramientas Compartidas}

Uso el término herramienta para señalar los aspectos metodológicos que se han desarrollado y se están desarrollando en la PC y en la PP y que constituyen aportes enriquecedores para ambas subdisciplinas. No hablaré de los métodos tradicionales de la psicología, de los cuales diariamente se sirve la PC, tales como encuestas con muestras representativas, entrevistas, especialmente las llamadas "profundas" y las semiestructuradas y focalizadas, y la observación. Me centraré más bien en los métodos que se consideran como propiamente comunitarios y en aquellos que, utilizados por la PP desde sus inicios, han sido tomados, con provecho, por la PC.

El método más importante en la $\mathrm{PC}$ es la investigación acción participativa (IAP). Este método surgió en otras ciencias sociales (sociología, antropología, educación popular) a mediados de los años 60 cuando, queriendo trabajar con la gente a su lado, Paulo Freire y los educadores y otros profesionales de las ciencias sociales que con él trabajaban (Rodríguez Brandão, 1981/1990), así como los sociólogos del grupo La Rosca, liderados por Fals Borda (2001, Hall, 2001, Park, 2001), modificaron en la práctica la idea lewiniana. Esa idea, vagamente descrita por Lewin en un artículo publicado póstumamente en 1948/1973, consistía en un movimiento de vaivén entre la planificación de acciones y su ejecución, que permitía analizar más rápidamente las respuestas de los sujetos, modificando los planes. Tanto Freire (1969, 1970/1997, 1988) como Fals Borda (1959, 1978, 1986, 2001), y quienes con ellos trabajaban, desarrollaron un método esencialmente participativo (aspecto no presente en la obra de Lewin), al incorporar en todas las fases de la investigación a los que antes se llamaba "sujetos", pero que dejaron de estar sujetados para tener voz, voto y veto en lo que se llevaba a cabo en sus comunidades. La PC incorpora a la IAP en los años 80 y desde entonces no la ha abandonado, enriqueciendo sus procedimientos desde la praxis. Actualmente este método es ampliamente usado por psicólogos/as comunitarios/as; una muestra interesante es la obra coordinada por Reason y Bradbury (2001), en la que colaboran investigadores de los cinco continentes.

El uso de los métodos biográficos tiene una notable tradición antropológica, algunos de cuyos productos, en los cuales construir la historia era una finalidad en sí misma, son famosos. Las biografías fueron muy populares en los años 60 y 70, al igual que los relatos de vida, utilizados como modo de reconstruir y de conservar aspectos culturales transmitidos por personas consideradas como modelos, que reunían en sus narraciones las características de una cultura. Asimismo, la psicología, en particular su rama política, utiliza este método desde el primer cuarto del siglo 20 en adelante. Son bien conocidos los trabajos de Lasswell (1930), de Erikson (1975) y, para citar a un solo autor contemporáneo, los de Winter (1996, 2003, 2004). Sobre la base de métodos biográficos y de técnicas como el análisis de contenido, revisado y adaptado, se desarrolla una corriente de la PP: la psicohistoria. A esos métodos se unen trabajos más recientes sobre análisis de discurso, realizados tanto en el ámbito psicopolítico como en el psicosocial comunitario, cuyo desarrollo e importancia ameritarían un espacio dedicado solo a ellos.

La PC toma estos métodos uniendo el giro participativo, el giro discursivo y el giro hermenéutico. La historia de vida tendrá, entonces, al menos dos autores: el o la psicóloga que la inicia y la persona biografiada que cuenta su vida, pero que también discutirá aspectos de la misma y participará en el análisis, muchas veces llevado a cabo con un equipo técnico o con otros biografiados y personas interesadas en su comunidad. El cruce de historias de vida y su discusión en reuniones de reflexión o en entrevistas participativas es una forma de enriquecer el relato, además de su valor como modo de recuperación de la memoria histórica (Fals Borda, 1986; Moreno, 1993, 1998; Moreno \& Luna, 2002).

Moreno (1998) utiliza la historia de vida en el campo comunitario, político y también en el estudio de las relaciones familiares. La historia de vida pasa a ser una construcción a cuatro manos entre el investigador y la persona biografiada, la cual se autoexami- 
na, a la vez que narra y discute su vida y el significado que atribuye a hechos en la misma. Ese método ha servido a Moreno para definir lo que ha llamado episteme popular, el modo de vida y el sentido de vida en los sectores más pobres de la sociedad venezolana, de donde provienen los narradores de vida. Descubre así un dédalo de relaciones, valores y significados que producen un modo de ser y que caracterizan a las personas que viven en situación de pobreza. Es un trabajo fascinante, pero se critica, no obstante, que Moreno haya centrado su visión en el mundo de vida popular de la pobreza, pues investigaciones realizadas en ese mismo sector, y simultáneamente en otros (medio y alto), arrojan datos coincidentes en algunos puntos (ciertamente no todos), por ejemplo, la presencia del matricentrismo, preponderante en la sociedad venezolana. Farías (2008) es otro autor que se inserta en esta línea de investigación y logra, a través de las historias de vida, describir y definir el altruismo en líderes comunitarios.

$\mathrm{Y}$ no se puede tratar este punto sin mencionar el análisis de narrativas comunitarias, otro método biográfico y discursivo, que Rappaport (1998, 2000) introduce en la PC estadounidense a mediados de los 90 y que constituye una forma de trabajar con la comunidad para, entre otros aspectos, analizar la identidad comunitaria y sus relaciones con las identidades individuales de otros miembros de la comunidad.

Algo común al uso de los métodos biográficos y discursivos es la introducción del giro hermenéutico, es decir, la búsqueda del significado para comprender la conducta y los procesos psicológicos unidos a ella, tarea fundamental de la psicología y absolutamente necesaria para quienes trabajamos en comunidades con las cuales compartimos un mismo idioma, aunque muchas veces usamos distinto lenguaje. La unión de la perspectiva hermenéutica con la participación es una relación feliz, pero no fácil de desarrollar (Farías, 2008; Hess, 2005; Moreno, 1993, 1998, 2003).

Un aporte de la PC es el modo creativo y participativo, con el cual, y siguiendo los principios de la IAP, se analizan los resultados de técnicas tradicionales, logrando que sean los grupos comunitarios quienes extraigan de los datos el mayor provecho y que a la vez se logren los objetivos de la investigación y acción. Es una manera en la cual tenemos certeza de que el método ha seguido al objeto y no viceversa. Pero no es tarea fácil, pues puede ser muy mal visto por algunos sectores académicos, además de que es necesario ser muy sistemático y riguroso a fin de organizar el procedimiento de manera comprensible para todos los sectores participantes.

Las presiones académicas han sido fuente inesperada de creatividad. En ese renglón entran las combinaciones de métodos mixtos que incorporan lo cualitativo y lo cuantitativo, no solo para trabajar en diferentes fases de la investigación y de la acción comunitarias, sino, incluso, haciendo combinaciones que podrían asustar a los ortodoxos de cualquiera de las dos tendencias mencionadas. Tal es el caso de los experimentos participativos cualitativos. Si esto parece algo imposible y aun escandaloso desde el punto de vista metodológico tradicional, que establecía un cisma entre lo cualitativo y lo cuantitativo -sería anatema-, pero no desde la perspectiva de la investigación comunitaria. El origen de ese método proviene de la necesidad de demostrar la validez y confiabilidad de intervenciones comunitarias que vayan más allá de la prueba de los hechos cumplidos. Así, buscando llenar requisitos convencionales necesarios para obtener un permiso, un apoyo o una subvención, se ha comprobado que la combinación enriquece el trabajo a realizar.

En términos tradicionales el modelo básico de experimento participativo cualitativo (Hernández, 2004; Sapene Chapellín, 2009) corresponde parcialmente a lo que Campbell y Stanley (1970) calificaban en 1966 como pre-experimental. Tales diseños constan de una medición previa, la introducción de una variable experimental y una medición posterior. Se comparan luego la primera y segunda mediciones y se deducen los resultados que pueden atribuirse a la condición introducida y controlada por el/la investigador/a. Lo interesante para quienes trabajamos desde un paradigma que busca los significados, que toma en cuenta la afectividad y que escucha las voces de las personas que participan en la investigación, es que la 
condición que se introduce es la participación en términos de IAP. Hernández (2004) y Sapene Chapellín (2009) han utilizado con precisión esta estrategia metodológica, obteniendo resultados de mucho interés.

Otro ejemplo muestra cómo en las encuestas con muestreo representativo, uno de los métodos más tradicionales de las ciencias sociales y cuya utilidad en la investigación y acción comunitaria es evidente en relación con -entre otros aspectos- la detección de necesidades, la recolección de datos demográficos o la medida de opiniones sobre algún hecho de interés comunitario, es posible introducir la participación de diversas maneras. Enseñar a las personas de la comunidad a construir y aplicar cuestionarios, acompañando a los/as agentes externos/as, es una de ellas. Eso incluye construir las preguntas del cuestionario junto con las personas de la comunidad interesadas en el proceso que se lleva a cabo (Montero, 2006a). Pero, aunque eso garantiza que las preguntas responderán a los intereses de la comunidad, sería solo instrumental y no estaría completa la participación si esas personas y muchas otras más no participasen activamente en el análisis, evaluación e interpretación de los resultados. Estas tres últimas tareas se llevan a cabo mediante la técnica de discusión crítica reflexiva (Montero, 1997) y de la problematización, que se ubica en lo que he llamado métodos para la conciencia (Montero, 2007c, 2009). Tausig (2002) presenta otro ejemplo interesante, al usar la perspectiva de redes para examinar las interacciones entre organizaciones grupales o "poblaciones" (en términos estadísticos) de organizaciones, para detectar "grietas" en los sistemas de salud mental dirigidos a la comunidad.

En general, la unión de métodos y su enriquecimiento participativo-reflexivo es cada vez más frecuente, además de ser útil en la psicología tanto comunitaria como política, y también de la salud, educacional, organizacional y en ciertas formas de trabajo clínico-comunitario (Montero \& Gonçalves de Freitas, 2009), ya que permite un enriquecimiento metodológico marcado por la participación que, al incorporar no solo las necesidades normativas sino también las sentidas y a quienes las sufren, logra acer- carse cada vez más a los problemas comunitarios y producir mejores y más efectivas respuestas a los mismos.

\section{Conclusión}

El intercambio justo entre organizaciones, comunidades e instituciones gubernamentales, con tratamiento cívico de las comunidades, genera modos de aplicar una $\mathrm{PC}$ que se reconoce como un modo alternativo de acción política. El proceso de formar y fortalecer la ciudadanía es un modo de ser políticos/as y de quehacer político. Es también un modo de desarrollar una sociedad civil fuerte, participativa, reflexiva y consciente de sus derechos y deberes, mediante procesos de concientización.

Orford (2008), comentando positivamente esta condición de la PC que se hace en muchos lugares de América Latina (Argentina, Bolivia, Brasil, Colombia, Costa Rica, Chile, Ecuador, Guatemala, Uruguay y Venezuela), usa al respecto una aguda expresión, al decir que es necesario contrarrestar el analfabetismo político de la psicología como disciplina científica. Y tiene razón, pues no se pueden dejar de lado los aspectos políticos de la PC.

Al mencionar los países que aparecen en el paréntesis del párrafo anterior, es necesario advertir que la PC no es uniformemente usada en todos los ámbitos de enseñanza y aplicación. La diversidad de tendencias y modelos coexiste, pero existen también líneas de influencia teórica y metodológica que continuamente se entrecruzan. Cuando se habla de una PC latinoamericana se señala un paradigma generado en esta parte del continente, expresado en producción escrita publicada nacional e internacionalmente. Podría así ocurrir que algún país no mencionado pudiese también tener producciones que podrían hacer suyo el comentario de Orford (2008).

No obstante, la aplicación de lo que llamamos métodos para la conciencia no es sencilla y suele ser blanco de los ataques de las tendencias autoritarias y totalitarias, que nada temen más que una sociedad pensante, reflexiva y activa. Quintal de Freitas (2003) en Brasil y Krause y Jaramillo (1998) y Krause (2002) en Chile, han señalado la 
existencia de una creciente desmovilización comunitaria (y, por ende, política), algo corroborado por Piper (2003) también en Chile, en relación con el debilitamiento de la crítica. Esto podría revertir el proceso de politización, en el buen sentido de la palabra, que es promovido desde el campo del desarrollo psicológico comunitario.

La incorporación en la $\mathrm{CP}$ de la perspectiva liberadora, corriente desarrollada en América Latina a partir de los años 90 y cuyo origen está en los escritos publicados por Martín-Baró entre 1983 y 1989, es parte de esa vocación política de la subdisciplina. Esta tendencia, iniciada en América Latina, tiene ya carácter global, pues se ha extendido a los cinco continentes, facilitando la coincidencia entre PP y PC, a la vez que muestra los beneficios de la unión entre ambas subdisciplinas (Montero, 2008; Montero \& Sonn, 2009; Nelson \& Prilleltensky, 2005; Reich et al., 2007). Cabe decir que en América Latina la mayoría de los trabajos comunitarios ha surgido en ámbitos multidisciplinarios e interdisciplinarios. En ello puede haber incidido que la formación teórica de la PC se haya construido por contribuciones tomadas de la filosofía, la sociología crítica, la educación popular y la antropología. Una conclusión general que podemos extraer de esta política comunitaria y comunidad politizada, en el sentido no partidario sino de ejercicio ciudadano, es que el área que se está integrando podría contribuir a la tan deseada transformación equitativa y liberadora de las sociedades que forman este continente.

\section{Referencias}

Acosta Pérez, E., Fonseca Lago, D., Malavé, S., Ortiz Torres, B., Rivera Ortiz, R., Villa, S. \& Walters, K. (2007). Debates contemporáneos en la psicología comunitaria: globalización, cambio social y empowerment. En E. Saforcada, N. Cervone, J. Castellá Sarriera, A. Lapalma \& M. De Lellis (Comps.), Aportes de la psicología comunitaria a problemáticas de la actualidad latinoamericana (pp. 253-272). Buenos Aires, Argentina: JVE.

Arango Cálad, C. (2006). Psicología comunitaria de la convivencia. Cali, Colombia: Universidad del Valle.

Campbell, D. \& Stanley, J. (1970). Diseños experimentales y cuasiexperimentales en la investigación social. Buenos Aires, Argentina: Amorrortu.
Campos, M. (1978). La dinámica de grupo en el desarrollo comunal (Tesis de Licenciatura no publicada), Facultad de Psicología, Universidad Central de Venezuela, Caracas, Venezuela.

Díaz Guerrero, R. (1972). Hacia una teoría históricobio-psico-socio-cultural del comportamiento humano. México, DF, México: Trillas.

Erikson, E. H. (1975). Historia personal y circunstancia histórica. Madrid, España: Alianza.

Eysenck, H. J. (1960/1964). Psicología de la decisión política (Título original: The psychology of politics). Barcelona, España: Ariel.

Fals Borda, O. (1959). Acción comunal en una vereda colombiana. Bogotá, Colombia: Universidad Nacional de Colombia.

Fals Borda, O. (1978). Por la praxis: el problema de cómo investigar la realidad para transformarla. En Simposio Mundial de Cartagena (Coord.), Crítica y política en ciencias sociales: el debate teoría y práctica (Vol. 1, pp. 209-249). Bogotá, Colombia: Punta de Lanza.

Fals Borda, O. (1986). Conocimiento y poder popular: lecciones con campesinos de Nicaragua, México y Colombia. Bogotá, Colombia: Siglo XXI.

Fals Borda, O. (2001). Participatory (action) research in social theory: Origins and challenges. En P. Reason \& H. Bradbury (Eds.), Handbook of action research: Participative inquiry \& practice (pp. 27 37). London, Reino Unido: Sage.

Farías, L. (2008). La comunidad en carne propia. Caracas, Venezuela: Universidad Central de Venezuela, Vicerrectorado Académico.

Flores Osorio, J. M. (2009). Praxis and liberation in the context of Latin American theory. En M. Montero \& C. C. Sonn (Eds.) Psychology of liberation: Theory and applications (pp.11-36). New York, NY: Springer. doi:10.1007/978-0-387-85784-8

Freire, P. (1969). La educación como práctica de la libertad. Montevideo, Uruguay: Tierra Nueva.

Freire, P. (1970/1997). Pedagogía del oprimido. México, DF, México: Siglo XXI.

Freire, P. (1988) ¿Extensión o comunicación? La concientización en el medio rural. Montevideo, Uruguay: Siglo XXI.

Fuks, S. (2007). Reflexiones acerca de la paradoja del empowerment. En E. Saforcada, N. Cervone, J. Castellá Sarriera, A. Lapalma \& M. De Lellis (Comps.) Aportes de la psicología comunitaria a problemáticas de la actualidad latinoamericana (pp. 19-52). Buenos Aires, Argentina: JVE.

Gissi, J. (1987). Identidad latinoamericana: psicología y sociedad. Santiago, Chile: Gráfica Andes.

Hall, B. L. (2001). I wish this were a poem of practices of participatory research. En P. Reason \& H. Bradbury (Eds.), Handbook of action research: Participative inquiry \& practice (pp. 171-178). London, Reino Unido: Sage.

Hernández, E. (1996). La comunidad como ámbito de participación. Un espacio para el desarrollo local. En E. Hernández (Coord.), La participación: ámbitos, retos y perspectivas (pp. 21-44). Caracas, Venezuela: CESAP.

Hernández, E. (2004). Metadecision: Training community leaders for effective decision-making. En M. Montero (Ed.), Leadership and organization for community prevention and intervention in Venezuela (pp. 53-70). Binghamton, NY: Haworth Press. 
Hess, J. Z. (2005). Scientists in the swamp: Narrowing the language-practice gap in community psychology. American Journal of Community Psychology, 35, 239-252. doi:10.1007/s10464-0053403-5

Holzkamp, K. (1985). Grundlegung der Psychologie [Fundamentos de la psicología]. Frankfurt, Alemania: Campus.

Irizarry, A. \& Serrano-García, I. (1979). Intervención en la investigación. Boletín de AVEPSO, 2(3), 6 21.

Krause, M. (2002). The institutionalization of community interventions in Chile: Characteristics and contradictions. American Journal of Community Psychology, 30, 547-570. doi:10.1023/ A:1015812118935

Krause, M. \& Jaramillo, A. (1998). Intervenciones psicológico comunitarias en Santiago de Chile. Santiago, Chile: Pontificia Universidad Católica de Chile.

Lane, R. E. \& Sears, D. O. (1964/1967) La opinión pública. Barcelona, España: Fontanella.

Lane, S. \& Sawaia, B. B. (1991). Psicología ¿ciencia o política? En M. Montero (Coord.), Acción y discurso. Problemas de psicología política en América Latina (pp. 59-85). Caracas, Venezuela: Ediciones Universitarias Venezolanas.

Lane, S. T. M. \& Sawaia, B. B. (1995) La psicología social comunitaria en Brasil. En E. Wiesenfeld \& E. Sánchez (Coords.), Psicología social comunitaria: contribuciones latinoamericanas (pp. 69-116). Caracas, Venezuela: Tropykos.

Lasswell, H. D. (1930) Psychopatology and politics. Chicago, IL: The University of Chicago Press.

Lazarsfeld, P. F., Berelson, B. \& Gaudet, H. (1944). The people's choice. New York, NY: Columbia University Press.

Lewin, K. (1948/1973). Resolving social conflicts. London, Reino Unido: Souvenir Press.

López-Sánchez, G. \& Serrano-García, I. (1995). Intervenciones de comunidad en Puerto Rico: el impacto de la psicología social comunitaria. En E. Wiesenfeld \& E. Sánchez (Coords.), Psicología social comunitaria: contribuciones latinoamericanas (pp. 219-248). Caracas, Venezuela: Tropykos.

Martín Baró, I. (1986). Hacia una psicología de la liberación. Boletín de Psicología (El Salvador), $22,219-231$.

Montero, M. (1984). Ideología, alienación e identidad nacional. Caracas, Venezuela: Universidad Central de Venezuela.

Montero, M. (1986). Political psychology in Latin America. En M. G. Hermann (Ed.), Political psychology (pp. 414-433). San Francisco, CA: Jossey-Bass.

Montero, M. (1987). A través del espejo: una aproximación teórica al estudio de la conciencia social en América Latina. En M. Montero (Coord.), Psicología política latinoamericana (pp. 163-202). Caracas, Venezuela: Panapo.

Montero, M. (1996). La participación: significados, alcances y límites. En E. Hernández (Coord.), Participación: ámbitos, retos y perspectivas (pp. 7-20), Caracas, Venezuela: CESAP.
Montero, M. (1997). Entre el deseo individual y la necesidad colectiva: el análisis de la reunión de discusión-reflexión en el trabajo psicosocial comunitario. En E. Wiesenfeld (Coord.), El horizonte de la transformación: acción y reflexión desde la psicología social comunitaria (AVESPO Fascículo 8, pp. 77-86). Caracas, Venezuela: Asociación Venezolana de Psicología Social.

Montero, M. (1998). Psychosocial community work as an alternative mode of political action: The construction and critical transformation of society. Community, Work \& Family, 1, 65-78. doi:10.1080/13668809808414698

Montero, M. (2000). Perspectivas y retos de la psicología de la liberación. En J. J. Vásquez O. (Comp.), Psicología social y liberación en América Latina (pp. 9-26). México, DF, México: Universidad Autónoma Metropolitana-Iztapalapa.

Montero, M. (2003a). Teoría y práctica de la psicología comunitaria: la tensión entre comunidad y sociedad. Buenos Aires, Argentina: Paidós.

Montero, M. (2003b). Relatedness as the basis for liberation. International Journal of Critical Psychology, 9, 61-74.

Montero, M. (2004). Relaciones entre psicología social comunitaria, psicología crítica y psicología de la liberación: una respuesta latinoamericana. Psykhe, 13(2), 17-28. doi:10.4067/S071822282004000200002

Montero, M. (2005). Para una ética de la liberación: la liberación del otro en la psicología. En N. Portillo, M. Gaborit \& J. M. Cruz (Comps.), Psicología social de la posguerra: teoría y aplicaciones desde El Salvador (pp. 410-435). San Salvador, El Salvador: UCA Editores.

Montero, M. (2006a). Le pouvoir et la psychologie politique de la communauté [El poder y la psicología política de la comunidad]. En A. Dorna \& J. M. Sabucedo (Coords.), Études et chantiers de psychologie politique [Estudios y proyectos de psicología política] (pp. 81-100). Paris, Francia: L'Harmattan.

Montero, M. (2006b). Hacer para transformar. El método en la psicología comunitaria. Buenos Aires, Argentina: Paidós.

Montero, M. (2007a). Fortalecimiento comunitario y formación ciudadana: un estudio psicosocial comunitario. En E. Saforcada, N. Cervone, J. Castellá Sarriera, A. Lapalma \& M. De Lellis (Comps.), Aportes de la psicología comunitaria a problemáticas de la actualidad latinoamericana (pp. 293-316). Buenos Aires, Argentina: JVE.

Montero, M. (2007b). The political psychology of liberation: From politics to ethics and back. Political Psychology, 28, 517-533. doi:10.1111/ j.1467-9221.2007.00588.x

Montero, M. (2007c). La problematización como aspecto crítico en el proceso de liberación. En I. Dobles Oropeza, S. Baltodano Arróliga \& V. Leandro Zúñiga (Coords.), Psicología de la liberación en el contexto de la globalización neoliberal: acciones, reflexiones y desafios (pp. 216-230). San José, Costa Rica: Universidad de Costa Rica.

Montero, M. (2008). An insider's look at the development and current state of community psychology in Latin America. Journal of Community Psychology, 36, 661-674. doi:10.1002/jcop.20241 
Montero, M. (2009). Methods for liberation: Critical consciousness in action. En M. Montero \& C. C. Sonn (Eds.), Psychology of liberation: Theory and applications (pp. 73-92). New York, NY: Springer. doi:10.1007/978-0-387-85784-8

Montero, M. \& Gonçalves de Freitas, M. (2009). Procedimientos y herramientas terapéuticas en contextos de pobreza: el método clínico-comunitario en construcción. En A. E. Hincapié Gómez (Coord.), Sujetos políticos y acción comunitaria: claves para una praxis de la psicología social y de la clínica social-comunitaria en América Latina (pp. 221-248). Medellín, Colombia: Universidad Pontificia Bolivariana.

Montero, M. \& Sonn, C. C. (Eds.) (2009). Psychology of liberation: Theory and applications. New York, NY: Springer. doi:10.1007/978-0-387-85784-8

Montero, M. \& Varas Díaz, N. (2007). Latin American community psychology: Development, implications, and challenges within a social change agenda. En S. M. Reich, M. Riemer, I. Prilleltensky \& M. Montero (Eds.), International community psychology: History and theories (pp. 63-98). New York, NY: Springer.

Moreno, A. (1993). El aro y la trama: episteme, modernidad y pueblo. Caracas, Venezuela: Universidad de Carabobo, Centro de Investigaciones Populares.

Moreno, A. (1998). Historia de vida de Felicia Varela. Caracas, Venezuela: Consejo Nacional de Investigaciones Científicas y Tecnológicas.

Moreno, A. (2003). Living and knowing: A participatoryhermeneutic approach to community psychology. International Journal of Critical Psychology, 9, 75-91.

Moreno, A. \& Luna, P. L. (2002). Buscando padre: historia de vida de Pedro Luis Luna. Caracas, Venezuela: Universidad de Carabobo, Centro de Investigaciones Populares.

Nelson, G. \& Prilleltensky, I. (Eds.) (2005). Community psychology: In pursuit of liberation \& well-being. New York, NY: Palgrave Macmillan.

Orford, J. (2008). Community psychology: Challenges, controversies and emerging consensus. Chichester, Reino Unido: John Wiley \& Sons.

Park, P. (2001). Knowledge and participatory research. En P. Reason \& H. Bradbury (Eds.), Handbook of action research: Participative inquiry \& practice (pp. 81-90). London, Reino Unido: Sage.

Patiño Pompa, T. (1985). Dinámica de grupos para la participación popular: entrenamiento de los facilitadores para la intervención (Tesis de Maestría no publicada), Universidad Central de Venezuela, Caracas, Venezuela.

Patiño Pompa, T. \& Millán Aguilar, Y. (1979). Análisis comparativo de dos investigaciones de campo de desarrollo comunitario (Tesis de Licenciatura no publicada), Universidad Central de Venezuela, Caracas, Venezuela.

Piper, I. (2003). The blurring of criticism: Notes on dissent. International Journal of Critical Psychology, 9, 125-142.

Prilleltensky, I. (2008). The role of power in wellness, oppression, and liberation: The promise of psychopolitical validity. Journal of Community Psychology, 36, 116-136. doi:10.1002/jcop.20225
Quintal de Freitas, M. F. (2003). Psychosocial practices and community dynamics: Meanings and possibilities for advancement from the perspective of social actors. International Journal of Critical Psychology, 9, 107-124.

Rappaport, J. (1981). In praise of paradox: A social policy of empowerment over prevention. American Journal of Community Psychology, 9, 1-25. doi:10.1007/BF00896357. Reproducido en T. A. Revenson, A. R. D' Augelli, S. E. French, D. L. Hughes, D. Livert, E. Seidman, M. Shinn \& H. Yoshikawa, (Eds., 2002), A quarter century of community psychology: Readings from the American Journal of Community Psychology (pp. 121-146). New York, NY: Kluwer Academic/ Plenum.

Rappaport, J. (1987). Terms of empowerment/ exemplars of prevention: Toward a theory for community psychology. American Journal of Community Psychology, 15, 121-148. doi:10.1007/ BF00919275

Rappaport, J. (1998). The art of social change: Community narratives as resources for individual and collective identity. En X. B. Arriaga \& S. Oskamp (Eds.), Addressing community problems: Psychological research and interventions (The Claremont Symposium of Applied Social Psychology, pp. 225-246). Thousand Oaks, CA: Sage.

Rappaport, J. (2000). Community narratives: Tales of terror and joy. American Journal of Community Psychology, 28, 1-24. doi:10.1023/ A:1005161528817

Reason, P. \& Bradbury, H. (2001). Handbook of action research: Participative inquiry \& practice. London, Reino Unido: Sage.

Reich, S. M., Riemer, M., Prilleltensky, I. \& Montero, M. (2007). International community psychology: Theory and histories. New York, NY: Springer.

Riger, S. (1993). What's wrong with empowerment. American Journal of Community Psychology, 21, 279-292. doi:10.1007/BF00941504

Rodríguez, P. E. (2006). La intervención clínica en contextos de pobreza y exclusión: algunas perspectivas. Revista Venezolana de Psicología Clínica Comunitaria, 6, 31-68.

Rodríguez Brandão, C. (1990). Pesquisar-participar [Investigar-participar]. En C. Rodríguez Brandão (Coord.), Pesquisa participante [Investigación participante] (2 ed., pp. 9-16). São Paulo, Brasil: Brasiliense.

Rodríguez-Ferreyra, A. (2009). Social policies in Uruguay: A view from the political dimension of community psychology. American Journal of Community Psychology, 43, 122-133. doi:10.1007/ s10464-008-9213-9

Salazar, J. M. (1983). Bases psicológicas del nacionalismo. México, DF: México: Trillas.

Sánchez, E. \& Wiesenfeld, E. (Coords.) (1995). Psicología social comunitaria: contribuciones latinoamericanas. Caracas, Venezuela: Tropykos.

Santi, B., Silva, I. \& Colmenares, F. (1978). Desarrollo comunal en la urbanización Urdaneta en Catia (Tesis de Licenciatura no publicada), Facultad de Psicología, Universidad Central de Venezuela, Caracas, Venezuela. 
Sapene Chapellín, A. (2009). The game of war: The liberating action of games in a context of political polarization. En M. Montero \& C. C. Sonn (Eds.), Psychology of liberation: Theory and applications (pp.173-191). New York, NY: Springer. doi:10.1007/978-0-387-85784-8

Serrano-García, I. \& López-Sánchez, G. (1994). Una perspectiva diferente del poder y el cambio social para la psicología social-comunitaria. En M. Montero (Coord.), Psicología social comunitaria (pp. 167-210). Guadalajara, México: Universidad de Guadalajara.

Shinn, M. (2007). Waltzing with a monster: Bringing research to bear on public policy. Journal of Social Issues, 63, 215-231. doi:10.1111/j.15404560.2007.00505.x

Smith, J. L. (2006). At a crossroad: Standing still and moving forward. American Journal of Community Psychology, 38, 23-25. doi:10.1007/s10464-0069058-z

Tausig, M. (2002). Detecting "cracks" in mental health service system: Application of network analytic techniques. En T. A. Revenson, A. R. D’Angelli, S. R. French, D. L. Hughes, D. Livert, E. Seidman, M. Shinn \& H. Yoshikawa (Eds.), Ecological research to promote social change: Methodological advances from community psychology (pp. 171186). New York, NY: Kluwer Academic/Plenum.

Vázquez Rivera, C. (2004). Refortalecimiento: un debate con el empowerment. Revista Interamericana de Psicología, 38, 41-51.
Watts, R. J., Griffith, D. M. \& Abdul-Adil, J. (1999). Sociopolitical development as an antidote for oppression: Theory and action. American Journal of Community Psychology, 27, 255-271. doi:10.1023/A:1022839818873

Winter, D. G. (1996). Personality: Analysis and interpretation of lives. New York, NY: McGrawHill.

Winter, D. G. (2003). Assessing leaders' personalities: A historical survey of academic research studies. En J. M. Post (Ed.), The psychological assessment of political leaders (pp. 11-38). Ann Arbor, MI: The University of Michigan Press.

Winter, D. G. (2004). Leader appeal, leader performance, and the motive profile of leaders and followers: A study of American presidents and elections. En J. T. Jost \& J. Sidanius (Eds.), Political psychology (pp.124-134). New York, NY: Psychology Press.

Zambrano, A. (2007). Participación y empoderamiento comunitario: rol de las metodologías implicativas. En A. Zambrano, G. Rozas, I. Magaña, D. Asún \& R. Pérez-Luco (Coords.), Psicología comunitaria en Chile: evolución, perspectivas y proyecciones (pp. 373-402). Santiago, Chile: Universidad de La Frontera/Universidad de Chile/Universidad de Santiago/Universidad de Valparaíso.

Zimmerman, M, A, (2000). Empowerment theory: Psychological, organizational, and community levels of analysis. En J. Rappaport \& E. Seidman (Eds.), Handbook of community psychology (pp. 43-63). New York, NY: Kluwer Academic/ Plenum. 